\title{
Natural Deodorants: A way towards sustainable cosmetics
}

HardikB. Bhatt ${ }^{\# 1}$, Dr. Nikunj B. Patel ${ }^{\# \prime}$

1,*Department of Microbiology,Smt. S. S. Patel Nootan Science \& Commerce College, SankalchandPatelUniversity,Visnagar-384 315

1'hardikbhatt86@yahoo.com,"niks17micro@gmail.com,

\section{ABSTRACT}

Deodorants and Antiperspirants are two of the most commonly used cosmetics, with millions of people applying them to their axilla regularly. Antiperspirants are used to minimize sweat production and deodorants are used to mask frequently, these two merged into a single product. Deodorant products inhibit the growth and activity of bacteria that degrade the apocrine gland in the armpit. Despite their effective antibacterial properties, common antibacterial agents such as triclosan and aluminum salts increase the risk of Alzheimer's disease, breast and prostate cancer, and contact dermatitis. As a consequence, plant extracts with antibacterial properties are useful. Herbal cosmetics products have grown in popularity among the general public. Herbal cosmetics are believed to have effectiveness and inherent acceptability due to their widespread use in everyday life while avoiding the negative effects associated with synthetic products. Herbal products are in high demand for primary health care in the developing world, not just because they are cheap, but also because they are more compatible with the human body and have fewer side effects, Natural deodorant and synthetic deodorant are of great importance. This study aims to review on beneficial applications of natural deodorant over synthetic ingredients of the formulation.

\section{Keywords:NaturalDeodorant,Antiperspirants,Body odor,Armpit,}

\section{Corresponding Author:Dr. Nikunj B. Patel}

\section{INTRODUCTION}

\section{The Physiology of Body Odor}

Skin is the largest organ that forms the boundary between the organism and also the environment (Reiger, 2000). It prevents skin dehydration, prevents the entry of weak foreign substances and microorganisms, cushions against mechanical shock, helps maintain vital signs, and transduces incoming stimuli (Reiger, 2000).

The bacterial degradation of precursors in sweat secretions is usually blamed for human armpit odor (Ferdenzi et al., 2020; Reinarz,2014). Age, sex, genetic conditions, environmental factors such as temperature or stress circumstances, grooming, and therefore the use of cosmetics may all lead to foulness by affecting the volume and consistency of secretions, as well as the types of bacteria present on the skin. A type of bacterial flora found on human skin that is usually considered an innocuous symbiont but can also be an opportunistic pathogen. traditional skin flora doesn't have an adverse effect on the skin except the assembly of odorous substances (Schlegel, 1993) bacterial flora on normal skin was found as predominantly gram-positive and it's assumed that the population of gram-negative bacteria has increased there with a rise in either temperature or humidity (Nipa, 2015). 
Bacterial skin flora is found to be commensal, symbiotic, or parasitic relative to the host, although alterations in host immune status are known to have a major impact. The kind of relationship established is usually inherent to bacteria (Chiller et al., 2001).

Sweat has no odor of its own. When it comes into contact with viruses and bacteria that are still present on the skin, foulness develops. Bacteria grow in moist environments, such as those found in your armpits (Pastor and Harper, 2012). When you sweat, bacteria break down those proteins in the sweat and convert them to acids. So the bacteria aren't the ones that stink. It's a by-product of the bacteria decomposing sweat(Inaba and Inaba,1992).

Apocrine perspiration is primarily made from proteins, lipids, carbohydrates, and nitrogen derivatives, and is, therefore, an excellent source for bacterial metabolism (Noble, 2004). The enzymes produced by the bacterial metabolism permit the components of sweat to rework into sub-products with a lower relative molecular mass, therefore becoming more volatile and producing an unpleasant odour(Franco, 2017).

Control of body odour involves the use of deodorants that act primarily depending on the mechanism of the action like the use of antimicrobial preparations to prevent the occurrence of bacteria flora(Halla et al.,2018) Antiperspirants such as aluminum salts are used to reduce the amount of perspiration. Perfumes or perfumed compounds are used to mask odors. Use of absorbent chemicals to neutralize odours (Piérard et al.,2003)

The most common bacteria present in armpits were Corynebacterium, Staphylococcus, Betaproteobacteria, Clostridiales, Lactobacillus, Propionibacterium, and Streptococcus. The bacterial inhabitants of armpits were found to be extremely diverse even within the small community of people; some were dominated by Corynebacterium species and some by Staphylobacter species. (Gao et al., 2010; Troccazet et al.,2015).The human armpit has a high biomass bacterial community, and up to date, studies have observed substantial interindividual variation in armpit bacteria, even relative to variation among individuals for other body habitats. One major explanation for this variation needs to do with the utilization of private hygiene products, particularly deodorants and antiperspirants (Urbanetet al., 2016)

\section{Deodorants and antiperspirants}

Aerosol, roll-on, creams \& wipes and sticks \& solids are the five main forms of deodorant and antiperspirant products. The market is divided into aerosol deodorants, roll-on deodorants, creams\& wipes deodorants, sticks \& solids deodorants, among others by deodorant product form(Plechner, 1972; Ficheux et al.,2015).

Deodorants and antiperspirants are two of the most popular cosmetic products, with many people applying them to their axilla on a daily basis. Deodorants are used to mask odors, while antiperspirants are used to reduce the amount of sweat produced. These two activities are often combined into a single product. The active ingredient in antiperspirants is sometimes aluminum-based, which reduces sweat by causing obstruction of the eccrine glands. Deodorants work by two different mechanisms (Abrutyn, 2015; Oliveira et al.,2021). Antimicrobial agents decrease the amount of bacteria that produce volatile odoriferous substances and perfume covers any odour that is produced. Recently, current zeolite minerals, which are sold as natural deodorants and antiperspirants in the form of aluminum or aluminum crystals (Laden, 1999). These items are available in solid crystal form. To prevent odour, the customer is advised to damp the crystals and add the product to the underarm region. About the fact that no study investigating the mode of action of such drugs has been conducted. Claims that the mineral salts create an environment during which bacteria cannot survive(Zirwas and Moennich, 2013).

Antiperspirants and deodorants are generally very safe products. However, these products have received much attention because the possible reason behind increasing rates of carcinoma and skin problems, with most hypotheses indicating the estrogenic properties of 
parabens because the main contributing factor. A spread of chemical antimicrobials is currently added to deodorants and antiperspirants to decrease the degree of odour-forming bacteria. These include antifreeze, triclosan, benzalkonium chloride and metal (e.g. Aluminum (Al)) salts (Kanlayavattanakul and Lourith,2011; Zirwas and Moennich,2013). The effectiveness of those chemical compounds depends on the kind of microbial flora present for every individual. Of concern, the security of the many chemical additives has yet to be determined and in some cases has been linked with serious health problems. Indeed, the common deodorant additive aluminum has been linked with a good range of negative health effects including degenerative neurological conditions (e.g. Alzheimer's disease, encephalopathy) (Shaw and Tomljenovic 2013; Exley, 1998) and cancer (Darbre et al., 2004), although the link to cancer has not been definitively proven. Studies have indicated that aluminum additives might also cause respiratory problems and cause anaphylaxis in susceptible individuals. Similarly, there are concerns about chronic exposure to triclosan, a standard bacterial growth inhibitor in many deodorants. Triclosan has been reported to cause a range of health problems including dermal irritation and allergies, although the authors of that study report relatively high levels of exposure are required for these effects to become evident (Bhargava and Leonard, 1996).

Deodorants play a serious role in allergic dermatitis caused by fragrances. Deodorants seem to possess an unfortunate composition of fragrance ingredients, resulting in allergic dermatitis. As deodorants are employed in sensitive areas of the body, it may well be argued that these sensitizing fragrance ingredients should either be avoided or utilized in lower concentrations in deodorants than in other forms of product. Natural alternatives that inhibit the expansion of odour-forming bacteria are desirable and should be more acceptable to consumers thanks to their natural origin and consumer perception of safety (Heisterbergetet al., 2011).

A deodorant is a product that is applied to the body to remove odor from bacterial breakdown in the armpits, feet, and other areas. Antiperspirants, a subgroup of deodorants, cause odor as well as sweating prevention by disrupting sweat glands. Antiperspirants are usually added to the underarms, although deodorants in the form of body sprays may be applied on the feet and other areas (Callewaert et al., 2014)

\section{Advantages of Natural deodorant over Synthetic}

Natural ingredients, such as plants, insects, microorganisms, and aquatic organisms, have been used in medicine since ancient times to alleviate and treat diseases. Plants have been used as drugs by humans for at least 60,000 years, according to fossil records (Parasuraman, 2018).

Natural deodorant is the modern trend in the field of beauty and fashion. These agents are gaining popularity as nowadays most people prefer natural products over synthetic materials for their personal care to enhance their beauty as these products supply the body with nutrients and enhance health and provide satisfaction as these are free from synthetic chemicals and have relatively less side-effects compared to the synthetic cosmetics (Joshi and Pawar,2015).

Antibacterial agents such as quaternary ammonium compounds like triclosan, aluminum salts, and aromatic odor-masking agents are now found in deodorant materials. Aluminum salts have an antibacterial benefit, but they can raise the risk of Alzheimer's disease, breast cancer, and prostate cancer. Many other antibacterial agents that have been found to be 
effective against skin bacteria are irritants or sensitizers. There's also the risk of developing resistance to common antibiotics. For the prevention of armpit odour, natural additives with antibacterial action against Staphylococci and Corynebacterium are an option. (Shahtalebi, 2013).

Nowadays, Deodorant products that are free from Parabens, aluminum, alcohol, and artificial preservatives, are getting very talked-about within the market due to the rising demand for safe, natural and organic products. Aim of this review to check different synthetic materials and natural materials against the axillary and foot odor-forming bacteria Corynebacteriumjeikeium,Staphylococcus epidermidis, Propionibacterium acnes and other odour causing bacteria with the aim of identifying safe and effective natural deodorant components (McManus et al.,2017).

Some study confirms that sufficient dosage of Soxhleted alcoholic extracts of Curry leaf, Methi Seeds \&Neem leaves not only effectively prevents body bad odor but also provides long lasting efficacy up to $48 \mathrm{hrs}$ (Vaibhav and Satish,2017), The methanolic leaf extract of Terminalia ferdinandianahas the ability to be used as a natural antibacterial ingredient in deodorant formulations. Due to the frequency at which deodorants are used, research is needed to ensure that their components do not aggregate and induce persistent toxicity. The low toxicity of Terminalia ferdinandiana leaf extracts, as well as their ability to prevent the growth of axillary and plantar malodour-producing bacteria, suggest that they may be used as deodorant ingredients (McManus et.al, 2017).

Agar well diffusion was used to test the effects of sage extract on sweat-decomposing bacteria. It was discovered that 1 percent $\mathrm{CO}_{2}$ sage extract inhibits Corynebacterium strains and $S$. epidermidis significantly. To check the in vitro antimicrobial effects of various sage extracts, researchers evaluated axillary deodorancy of dichloromethane sage extract in a silicone-based stick formulation (Shahtalebi et al.,2013)

The health effects of antiperspirants are a matter of dispute regarding their extent. A small percentage of people are allergic to aluminium and may experience contact dermatitis when exposed to aluminium-containing deodorants. Deodorant crystals containing synthetically made potassium alum were found to be a weak irritant to the skin. Natural Deodorants are a great way to avoid parabens, aluminum, and neuro-toxins found in commercial deodorants and antiperspirants. Because of above reason herbal antimicrobial agents are used in the formulation of deodorant stick. Experiment was performed by selecting the plant oil of Eugena caryophyllus which was reported to have antibacterial activity (Debnath et al.,2011)

\section{Conclusion}

Consumers are increasingly searching for cosmetics that have a lower environmental impact. The demand for natural cosmetics is increasing, as it is widely accepted that these ingredients are safe and free of side effects. In the personal care industry, natural deodorant is becoming more common. All of this has occurred as a result of the widespread use of synthetic-based materials, synthetic additives, organic dyes, and their derivatives; their manufacture and use pose a health risk to humans, with a variety of side effects contributing to a variety of diseases. 


\section{Reference}

1. Abrutyn ES. antiperspirants and Deodorants. Cosmetic Dermatology: Products and Procedures. 2015 Oct 8:160.

2. Alnuqaydan A. Determination of Cancer Induction and Cell-Killing Potential of Beauty Products and Personal Care Products Using Human Skin Cells (Doctoral dissertation, Flinders University).

3. Baranoski S, Ayello EA, Tomic-Canic M, Levine J. Skin: an essential organ. Wound Care Essentials: Practice Principles. 3rd ed. Philadelphia, PA: Lippincott Williams \& Wilkins. 2012.

4. Bhargava HN, Leonard PA. Triclosan: applications and safety. American journal of infection control. 1996 Jun 1;24(3):209-18.

5. Callewaert C, Hutapea P, Van de Wiele T, Boon N. Deodorants and antiperspirants affect the axillary bacterial community. Archives of dermatological research. 2014 Oct 1;306(8):701-10.

6. Chiller K, Selkin BA, Murakawa GJ. Skin microflora and bacterial infections of the skin. InJournal of Investigative Dermatology Symposium Proceedings 2001 Dec 1 (Vol. 6, No. 3, pp. 170-174). Elsevier.

7. Darbre PD, Aljarrah A, Miller WR, Coldham NG, Sauer MJ, Pope GS. Concentrations of parabens in human breast tumours. Journal of Applied Toxicology: An International Journal. 2004 Jan;24(1):5-13.

8. Debnath S, Babu MN, Kusuma G. Formulation and evaluation of herbal antimicrobial deodorant stick. Research Journal of Topical and Cosmetic Sciences. 2011;2(1):21.

9. Exley C. Does antiperspirant use increase the risk of aluminium-related disease, including Alzheimer's disease?. Molecular medicine today. 1998 Mar 1;4(3):107-9.

10. Ferdenzi C, Richard Ortegón S, Delplanque S, Baldovini N, Bensafi M. Interdisciplinary challenges for elucidating human olfactory attractiveness. Philosophical Transactions of the Royal Society B. 2020 Jun 8;375(1800):20190268.

11. Ficheux AS, Wesolek N, Chevillotte G, Roudot AC. Consumption of cosmetic products by the French population. First part: frequency data. Food and Chemical Toxicology. 2015 Apr 1;78:159-69.

12. Halla N, Fernandes IP, Heleno SA, Costa P, Boucherit-Otmani Z, Boucherit K, Rodrigues AE, Ferreira IC, Barreiro MF. Cosmetics preservation: a review on present strategies. Molecules. 2018 Jul;23(7):1571.

13. Heisterberg MV, Menné T, Andersen KE, Avnstorp C, Kristensen B, Kristensen O, Kaaber K, Laurberg G, Henrik Nielsen N, Sommerlund M, Thormann J. Deodorants are the leading cause of allergic contact dermatitis to fragrance ingredients. Contact Dermatitis. 2011 May;64(5):258-64.1

14. Inaba M, Inaba Y. Human body odor. etiology, treatment, and related factors. 1992:1992.

15. Joshi LS, Pawar HA. Herbal cosmetics and cosmeceuticals: An overview. Nat Prod Chem Res. 2015 Feb 16;3(2):170.

16. Laden K. Deodorant Technology Trends from. Antiperspirants and Deodorants. 1999 Jan 4:357.

17. McManus K, Wood A, Wright MH, Matthews B, Greene AC, Cock IE. TerminaliaferdinandianaExell. extracts inhibit the growth of body odour-forming bacteria. International journal of cosmetic science. 2017 Oct;39(5):500-10.

18. Nipa KK. Human Skin Bacteria: Innocuous Symbiotic Association, Pathogenic Action and Antibiotic Effect.

19. Noble WC, editor. The skin microflora and microbial skin disease. Cambridge University Press; 2004 Nov 11. 
20. Oliveira EC, Salvador DS, Holsback V, Shultz JD, Michniak-Kohn BB, Leonardi GR. Deodorants and antiperspirants: identification of new strategies and perspectives to prevent and control malodor and sweat of the body. International Journal of Dermatology. 2021 Mar 1 .

21. Parasuraman S. Herbal drug discovery: challenges and perspectives. Current Pharmacogenomics and Personalized Medicine (Formerly Current Pharmacogenomics). 2018 Apr 1;16(1):63-8.

22. Pastor DK, Harper DS. Treating body odor in primary care. The Nurse Practitioner. 2012 Mar 13;37(3):15-8.

23. Piérard GE, Elsner P, Marks R, Masson P, Paye M. EEMCO guidance for the efficacy assessment of antiperspirants and deodorants. Skin Pharmacology and Physiology. 2003; 16(5):324-42.

24. Plechner SO, Balsam MS, Sagarin E. Antiperspirants and deodorants. Cosmetics, Science and Technology. 1972 Jun 16;2:373-415.

25. Reiger MM. Harry's Cosmeticology, Volumes I-II. Chemical Publishing Company; 2000

26. Reinarz J. Past scents: Historical perspectives on smell. University of Illinois Press; 2014 Mar 30.

27. Schlegel. H.G. 1993. General microbiology. 7th edition. Cambridge University Press. p. 655.

28. Shahtalebi MA, Ghanadian M, Farzan A, Shiri N, Shokri D, Fatemi SA. Deodorant effects of a sage extract stick: Antibacterial activity and sensory evaluation of axillary deodorancy. Journal of research in medical sciences: the official journal of Isfahan University of Medical Sciences. 2013 Oct;18(10):833.

29. Shaw CA, Tomljenovic L. Aluminum in the central nervous system (CNS): toxicity in humans and animals, vaccine adjuvants, and autoimmunity. Immunologic research. 2013 Jul;56(2):304-16.

30. Troccaz M, Gaïa N, Beccucci S, Schrenzel J, Cayeux I, Starkenmann C, Lazarevic V. Mapping axillary microbiota responsible for body odours using a culture-independent approach. Microbiome. 2015 Dec;3(1):3.

31. Urban J, Fergus DJ, Savage AM, Ehlers M, Menninger HL, Dunn RR, Horvath JE. The effect of habitual and experimental antiperspirant and deodorant product use on the armpit microbiome. PeerJ. 2016 Feb 2;4:e1605.

32. VaibhavKhandare, Dr.SatishSakhawade,Formulation\& long lasting efficacy Evaluation of Herbal based Deodorant up to 48 hours by Sniff Test Method, International journal of research and finance ,2017 April,(Vol .7 issue 4,pp 210 to 216)

33. Zirwas MJ, Moennich J. Antiperspirant and deodorant allergy: diagnosis and management. The Journal of clinical and aesthetic dermatology. 2008 Sep;1(3):38. 